\title{
Image-Based Modelling Restitution: Pipeline for Accuracy Optimisation
}

\author{
Marco Limongiello \\ Lucas Matias Gujski
}

Abstract

The paper presents an innovative approach to support survey methods by applying Al algorithms to improve the accuracy of point clouds generated from UAV imagery. The work analyses different photogrammetric accuracy parameters in a first step, such as reprojection error and the intersection angle between homologous rays, verifying that a single parameter is enough to evaluate the accuracy of the photogrammetric restitution. Therefore, some of the calculated parameters were analysed through a Self-Organizing Map (SOM) to reach a compromise between the value of the variables analysed and the noise reduction associated with the 3D model definition. In the case study, it has been observed that the parameter that most influences the noise in the photogrammetric point clouds is the intersection angle.

Keywords

UAV, photogrammetry, accuracy, point cloud, SOM.

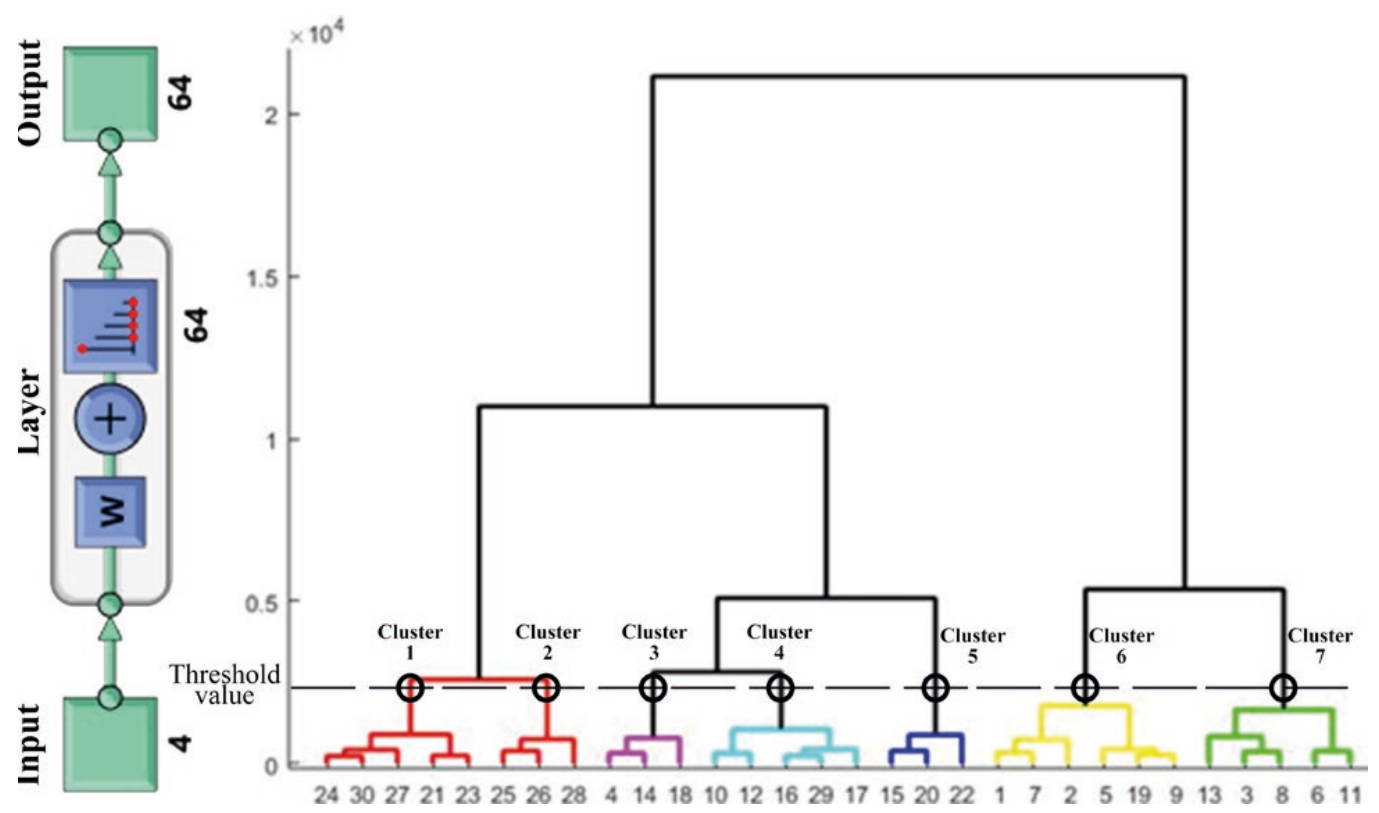




\section{Introduction}

During the past ten years, the application of photogrammetry in digital 3D recording has grown greatly. In fact, due to the development of the ComputerVision technology and the Structure of Motion (SFM) algorithms, the processing time of the mostly automatised photogrammetric workflow has accelerated exponentially, solving what was once a well-known weakness [Falkingham 20I2, pp. I-I5]. In addition, thanks to their technological development, the Unmanned Aerial Vehicles (UAV) have become easier to pilot and more reliable, a fact that indirectly promotes the growth of the photogrammetric applications, especially at a medium and large scale. Due to the acquisition speed and the transportability of the vehicle, the technology is indeed very versatile, allowing these instruments to be used in different applications [Fernández-Hernandez 20 I4, pp. I28-|45].

A topic debated in the scientific world is the evaluation of the accuracy of point clouds, particularly of the Tie points (TP), generated by processing either UAV or terrestrial, also known as Close Range images. A low accuracy of the model may, in fact, invalidate the high resolution of the data, thereby vitiating the graphic scale and derived products (i.e., plan, section, elevation). Especially in the Cultural Heritage sector, the accuracy of the metric system must be evaluated too, in order to avoid 'incorrect' documentation from the metric perspective.

A sparse point cloud just composed ofTPs is the first stage to obtain a complete 3D model; however, it is obvious that there are lower quality TPs. It is therefore appropriate to delete them to not affect the results of the subsequent steps. Most photogrammetric software offers the possibility to filter the TPs only based on an estimation of the Reprojection Error (RE) parameter associated with each TP [Barba 2019, pp. I-19].

In this work we propose an algorithm and a clustering method based on the Self-Organizing Maps (SOM), a type of neural network trained using unsupervised learning to produce a representation (usually a two-dimensional map) of the input data space.

SOM was needed to select the groups of points with similar characteristics that produce more noise, so to obtain a TP cloud containing just the points with greater accuracy. The subdivision of the TP cloud into different clusters made possible its discretization into different accuracy classes, which can be activated or not according to the level of detail to be pursued.

\section{Case Study and Data Acquisition}

The case study considered to develop this work is the Norman-Swabian Castle of Vibo Valentia, surveyed using a UAV in 2017. Currently, the castle hosts the Archaeological Museum 'Vito Capialbi' and the provincial offices of the Department of Cultural Heritage. The UAV system used for this application is a DII Inspire with a net weight of the sensor of about $3 \mathrm{~kg}$. The installed Zenmuse $X 3$ camera has a sensor of $1 / 2.3^{\prime \prime}$, with a resolution of 12 megapixel (4096x2160 pixels, $6.17 \times 4.55 \mathrm{~mm}$, Pixel Size of 1.56 Micron), a focal length of $4 \mathrm{~mm}$ and a Field of View - FOV $94^{\circ}$. The images were acquired through two different modalities: firstly, 106 nadir images were acquired with an automatic double-grid flight plan at a relative altitude of $25 \mathrm{~m}$ from the castle's inner forecourt, taking into account an overlap and a sidelap of $80 \%$ and $60 \%$ respectively. Subsequently, in manual mode, 950 oblique images with different inclinations $\left(30^{\circ}-60^{\circ}\right)$ were acquired, with the main objective to reconstruct the external and internal facades of the castle. The GSD, from the calculated data available, has been estimated on average considering also the oblique images at $1.3 \mathrm{~cm} / \mathrm{px}$, while considering only the nadiral images the GSD is about $1 \mathrm{~cm} / \mathrm{px}$. To support the photogrammetric project, 10 Ground Control Points (GCPs) were measured to georeference and assess the accuracy of the generated 3D model and orthophotos. The GCPs were materialized on the ground, using photogrammetric targets $(30 \times 30 \mathrm{~cm})$ and topographic nails. The GNSS survey refers to the Italian geodetic and cartographic system UTM/ETRFOO and was used the technique network Real Time Kinematic (nRTK). The instrumentation used to measure each target consists of an antenna with a built-in receiver of the Geomax Zenith 25. The accuracy obtained in planimetry is, on average, subcentimetric, while it is around $2.5 \mathrm{~cm}$ in altimetry. 


\section{Developed Methodology and Quality Features}

In order to generate a 3D model of the surveyed area, the Agisoft Metashape software was used. The following parameters have been set to process the point cloud: in the 'Align Photos' stage, accuracy = High (original image), while the calculation of Keypoints and TPS have been programmed as unlimited. The professional version of Agisoft Metashape uses Python 3 as scripting engine and has therefore better interfaces for the purposes of extracting the very inherent reconstruction accuracy parameters that we wanted to export. The following quality features were examined in detail.

- Reprojection Error: the geometric error, corresponding to the image distance between the projected point and the measured point [James 2017, pp. $5 \mathrm{I}-66$ ], is defined as Reprojection Error (RE). It is used to measure how accurately an estimated 3D point recreates the true projection of the point. The frequency distribution of the REs that better fit the data was analysed using Matlab (i.e. the Statistics toolbox). The distribution was used to exclude the external values, that are considered outliers at a selected experimental threshold. The algorithm implemented in the Python environment has been used to remove the 3D point above the threshold of the statistical significance coefficient $(\alpha)$.

- Angle between homologous points and Average distance: by estimating the angle between the two projection lines (called the 'intersection angle'), the Base/Height ratio (one of the parameters that have the greatest impact on the accuracy of the photogrammetric project [Kraus 2007]) is analysed. The photogrammetry software used does not give the value of this angle in the output, so we implemented a Python algorithm. The intersection angle calculation was executed using all the image pairs that contain the $i$-th TP; once the intersection angle for each pair had been calculated, it was possible to finally determine the average intersection angle between the $\mathrm{n}$ images that contain the point, removing the extreme values. Using the number of images and distances already calculated, the average distance between the i-th TP and the $\mathrm{n}$ images, was calculated. Finally, with each Tie Point extracted, the proposed method associates the average distance and the average angle value obtained. The whole process is implemented in Python.

- Image redundancy: this parameter is the number of photogrammetric shots implemented within the SfM process, for the reconstruction of the i-th TP in 3D space. With the same other parameters of photogrammetric accuracy, it is assumed that as the image redundancy increases, the metric quality of the TP point cloud improves.

-SOM - Self-organizing map [Kohonen 200I]: is an artificial neural network machine learning technique [Teruggi 2020, pp. I-27] usually used for visualization and analysis of high-dimensional data. Moreover, SOM is used for data clustering. Self-organizing maps can be combined with dimensionality reduction methods as multi-dimensional scaling [Kurasova 201 I, pp. II5-134]. The number of clusters to be brought into the accuracy analysis is extracted from the graphical representation of the dendrogram. A dendrogram (in first page) is a diagram representing a tree that shows the hierarchical relationship between object and used to visualise the similarity in the clustering process. In clustering techniques, the dendrogram is used to provide a graphic representation.

\section{Analysis of Individual Accuracy Parameters}

A very noisy standard portion, $50 \mathrm{~cm}$ wide, containing vertical walls and the inner yard, was analysed for the purpose of the study. The first parameter considered is RE. The Weibull distribution is the one best-fitting the interpolated data, therefore it was chosen to estimate the characteristic factors of the distribution. The distribution study was used to determine the threshold values, in order to remove the points with associated RE values above the estimated threshold values, more specifically those above the $99,95,90$ percentiles.

It is possible to observe (fig. I) how the filtering of the point cloud by analysing the RE and the statistical approach does not generate a good degree of filtering for the section under consideration; in fact, the procedure removes some spots mostly scattered, but it does not lead to great advantages in noise reduction. However, most of the isolated points were not filtered out. 
A better result for noise reduction is obtained by filtering the point cloud according to the average angle of intersection, calculated for each Tie-Point, and then analysing the acquisition geometry.

Excluding small average angles of intersection, we have obtained surfaces that are much more realistic and less noisy. Pushing the filter too much, setting high angle values as threshold, compromises the amount of data necessary for the representation; in fact, by setting an average angle over $20^{\circ}$ as a threshold, large quantities of points belonging to the vertical walls are removed (fig. I).

As for the parameter of the average distance between the i-th camera and the TPi, it is a parameter that does not affect the noise. It was also considered to take into account the density of the point cloud: high distances do not allow high GSDs, and therefore not very dense point clouds and cloud sections.

\section{SOM Analysis and Conclusion}

It was decided to experiment with a SOM method in order to take into account not only a single accuracy parameter but all the measured parameters at the same time. Using the Matlab Neural Network Clustering App, 4 values was used as input, which were the RE, number of images, average angle and average distance. The SOM Layer loaded with an $8 \times 8$ network and 100 epochs. The graphical representation of the dendrogram is used to select the number of clusters for the accuracy analysis. Once the dendrogram is created, 7 categories can be identified. It was decided to divide these 7 categories into 4 groups (fig. 2): 3 accuracy categories (High, Medium and Low) and a noise category. In order to reorganize the clustering of these accuracy levels, we analysed the average angle, the parameters of the maximum and minimum angles, and the number of cameras.

The established clusters 3 and 4 , which have the widest mean angle, maximum angle and minimum angle, are classified in this new classification as the High Class. Cluster 2 has been set as the Medium Class, clusters I and 7 as the Low Class, and finally clusters 5 and 6 as the Noise Class, with lower intersection values.

Analysing the cluster of the point cloud we overlapped (fig. 2) the 'High Layer Accuracy' containing the highest angle intersection with the 'Noise Cluster' containing the lowest value. It can be seen that the 'High Layer Accuracy' turns out to be the best fitting point set for vertical walls and ground.

The work shows that filtering the point cloud by evaluating the RE and using a statistical approach individually does not produce good filtering quality; in fact, even with high percentiles, some outliers were still not filtered out. Filtering according to the average intersection

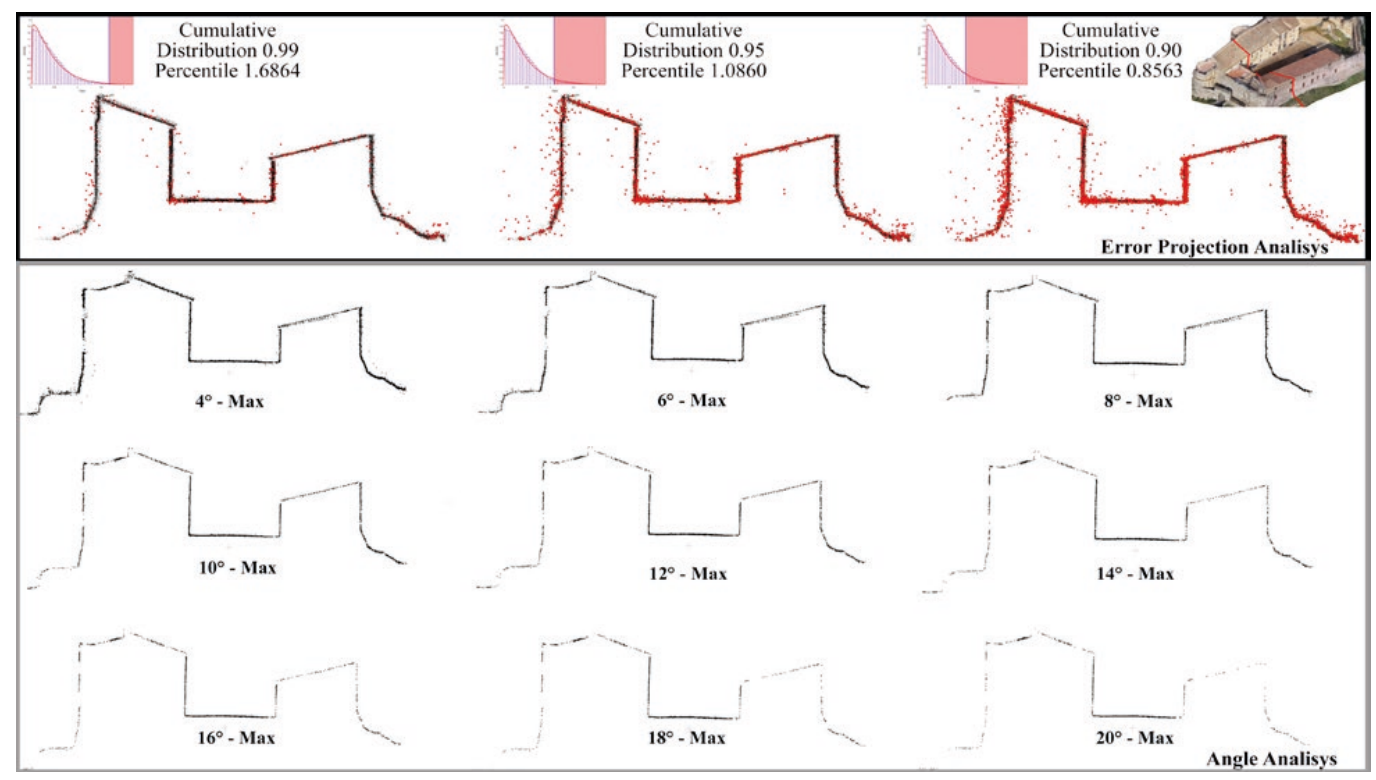




\begin{tabular}{|c|c|c|c|c|c|c|}
\hline Cluster & Error_Proj(Px) & Num Img & Angle_min $\left({ }^{\circ}\right)$ & Angle max $\left({ }^{\circ}\right)$ & Angle_Average $\left({ }^{\circ}\right)$ & Accuracy \\
\hline 1 & 0,39 & 53 & 0,5 & 28,3 & 6,5 & LOW \\
\hline 2 & 0,51 & 72 & 0,5 & 28,0 & 10,2 & MEDIUM \\
\hline 3 & 0,48 & 120 & 6,3 & 64,6 & 14,4 & \multirow{2}{*}{ HIGH } \\
\hline 4 & 0,20 & 89 & 7,4 & 45,1 & 18,1 & \\
\hline 5 & 0,20 & 64 & 0,1 & 30,1 & 1,1 & NOISE \\
\hline 6 & 0,26 & 30 & 0,1 & 18,3 & 2,1 & LOW \\
\hline 7 & 0,36 & 55 & 0,2 & 25,0 & 6,4 & LOW \\
\hline
\end{tabular}
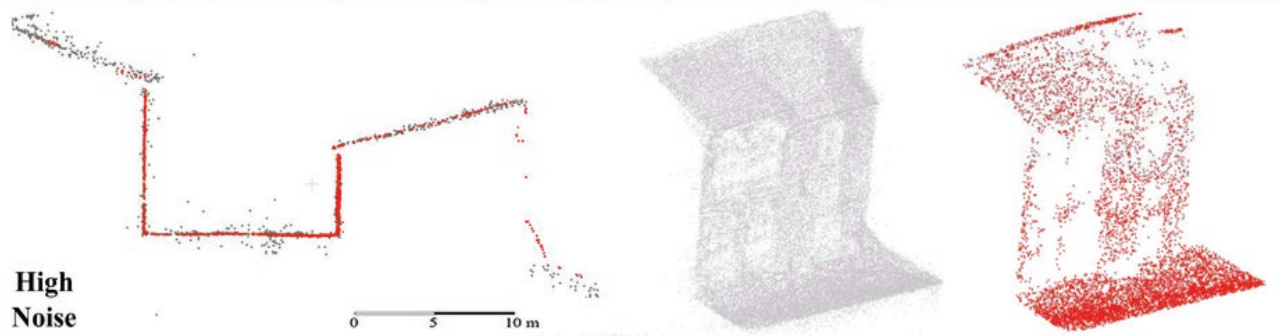

angle parameter alone produces a better result for 'noise' reduction. However, filtering through high angles can compromise the data density and cause, as a result, loss of information. With the SOM approach, using all the parameters calculated at the same time and the clustering process, the value of the RE does not change significantly. By analysing the number of images forming the cluster, the greater the number, the greater the noise reduction obtained. Using the cluster angle analysis, we can conclude that the clusters with the highest base-to-height ratio are considered to have the highest noise reduction. Generally, a trend can be defined, i.e. clusters with higher mean angles generate TP sections with less noise. From the analysis above, the parameter that most influences the noise in a TP point cloud is the mean intersection angle. Therefore, we can conclude that the advantages of using Al, in particular SOM, a relatively simple method applicable to point clouds, has allowed a fast clusterization, from which TPs can be selected with different accuracies, depending on the subsequent purposes.

\section{References}

Barba Salvatore, Barbarella Maurizio, Di Benedetto Alessandro, Fiani Margherita, Gujski Lucas, Limongiello Marco (2019). Accuracy Assessment of 3D Photogrammetric Models from an Unmanned Aerial Vehicle. In Drones, 3, pp. I - I 9

Falkingham Peter (2012). Acquisition of high resolution three-dimensional models using free, open-source, photogrammetric software. In Palaeontologia Electronica, I 5. I, pp. I- 15.

Fernandez-Hernandez Jesus, González-Aguilera Diego, Rodríguez-Gonzálvez Pablo, Mancera-Taboada J. (20I4). ImageBased Modelling from Unmanned Aerial Vehicle (UAV) Photogrammetry: An Effective, Low-Cost Tool for Archaeological Applications. In Archaeometry, 57, pp. $128-145$.

James Mike R., Robson Stuart, d'Oleire-Oltmanns Sebastian, Niethammer Uwe (2017). Optimising UAV topographic surveys processed with structure-from-motion: Ground control quality, quantity and bundle adjustment. In Geomorphology, 280, pp. 5 I -66.

Kohonen Teuvo (200I). Self-Organizing Maps. 3rd edition. Berlin: Springer-Verlag.

Kraus Karl (2007). Photogrammetry: geometry from images and laser scans. 2nd edition. Berlin:Walter de Gruyter.

Kurasova Olga, Molyte Alma (20II). Quality of Quantization and Visualization of Vectors Obtained by Neural Gas and SelfOrganizing Map. In Informatica, 22, pp. I I 5-134.

Teruggi Simone, Grilli Eleonora, Russo Michele, Fassi Francesco, Remondino Fabio (2020). A Hierarchical Machine Learning Approach for Multi-Level and Multi-Resolution 3D Point Cloud Classification. In Remote Sensing, 12, pp. I-27.

\section{Authors}

Marco Limongiello, Dept. of Civil Engineering (DICIV), University of Salerno, mlimongiello@unisa.it Lucas Matias Gujski, Dept. of Civil Engineering (DICIV), University of Salerno, Igujski@unisa.it 
